\section{Cureus}

\title{
Sporadic Erythromelalgia Associated with a Homozygous Carrier of Common Missense Polymorphism in SCN9A Gene Coding for NaV1.7 Voltage-gated Sodium Channel
}

\author{
Piotr K. Janicki ${ }^{1}$, Victor Ruiz-Velasco ${ }^{1}$, Sanjib Adhikary ${ }^{1}$ \\ 1. Anesthesiology and Perioperative Medicine, Penn State Milton S. Hershey Medical Center, Hershey, USA
}

$\square$ Corresponding author: Piotr K. Janicki, pjanicki@pennstatehealth.psu.edu

Disclosures can be found in Additional Information at the end of the article

\section{Abstract}

A 68-year-old female with a history of sporadic type and presumably secondary erythromelalgia with chronic intractable pain presented for foot surgery. The procedure was performed with combined general anesthesia and regional anesthesia consisting of the placement of a popliteal pain catheter for postoperative pain management. Subsequent whole-genome sequencing revealed that the patient was a homozygous carrier of the common missense mutation in the SCN9A gene coding for voltage-gated sodium channel (NaV1.7) - dbSNP rs6746030 (R1150W). The occurrence of this single nucleotide polymorphism (SNP) was previously suggested not to be associated with erythromelalgia but rather thought to be part of quantitative changes in the pain threshold in different cohorts of patients. The placement of the pain catheter, although controversial in patients with erythromelalgia, provided effective postoperative pain relief without any side effects.

Categories: Anesthesiology, Genetics, Pain Management

Keywords: erythromelalgia, pain management, single nucleotide polymorphism, nav1.7 sodium channels, whole exome sequencing

\section{Introduction}

Erythromelalgia is a disabling chronic pain condition characterized by chronic pain and erythema of the extremities, particularly feet, which is exacerbated by warmth and relieved by cooling. The erythromelalgia can be either secondary (mostly due to myelodysplasia), primary genetic (due to rare mutations in the SCN9A gene coding for NaV1.7 voltage-gated sodium channels), or sporadic (idiopathic), when no obvious etiology can be found [1-2]. We report here the identification of a homozygous carrier for the common missense single nucleotide polymorphism (dbSNP rs6746030) within the SCN9A gene in a chronic pain patient previously diagnosed with secondary erythromelalgia due to minimal myelodysplasia of the bone marrow and undergoing a surgical procedure for the lower extremities.

Received 04/09/2019

Review began 04/14/2019 Review ended 04/29/2019 Published 05/02/2019

\section{Copyright 2019}

Janicki et al. This is an open access article distributed under the terms of the Creative Commons Attribution License CC-BY 3.0., which permits unrestricted use, distribution, and reproduction in any medium, provided the original author and source are credited.

\section{Case Presentation}

The reported patient is a 68-year-old white female seen in the preoperative evaluation clinic for planned multiple toe amputations and tenotomies of the right foot due to osteomyelitis. The patient's medical history included chronic pain syndrome as a consequence of secondary erythromelalgia. She also presented with a history of severe pain in her bilateral hands and feet, as well as a history of marked erythema of the palms of her hands and soles of her feet.

Other medical conditions included gastroesophageal reflux disease (GERD), chronic low back pain, previous atrial fibrillation episodes, and large and small fiber peripheral neuropathy of unknown etiology, causing occasional wrist drop. The chronic (several years) pain with more recent (three to four years) swelling and painful erythema of both feet prompted her to seek multiple medical consults several years ago. This led to bone marrow biopsy with a suspicion of erythromelalgia, which indicated mildly hypercellular bone marrow (70\%) with maturing trilineage hematopoiesis, including minimal, normoblastic erythroid hyperplasia. The cytogenetic results, including fluorescence in situ hybridization assay, BCR/ABL1 gene sequence, and JAK gene V617F mutation studies, were all negative. The hematologic profile from peripheral blood indicated

\section{How to cite this article}

Janicki P K, Ruiz-Velasco V, Adhikary S (May 02, 2019) Sporadic Erythromelalgia Associated with a Homozygous Carrier of Common Missense Polymorphism in SCN9A Gene Coding for NaV1.7 Voltagegated Sodium Channel. Cureus 11(5): e4587. DOI 10.7759/cureus.4587 
persistent anemia with an elevated red cell distribution width but a normal platelet number. The hematology consultant diagnosed the patient condition (in connection with clinical symptoms) as secondary erythromelalgia.

Because of the intractable chronic pain, the patient's medication list included multiple medications associated with pain treatment consisting of oxycodone (40-60 mg daily), tramadol (200 mg daily), pregabalin (300 mg daily), nortriptyline (100 mg daily), aspirin (325 mg daily), ibuprofen $800 \mathrm{mg}$ daily, and mexiletine ( $600 \mathrm{mg}$ daily). In addition, the patient applied four daily patches of $5 \%$ topical lidocaine to the feet for 12 hours.

Due to the patient's extensive history of intractable pain (including exacerbation of pain symptoms postoperatively with previous surgeries), we discussed the combined regional anesthesia, with general anesthesia as the anesthetic technique. For general anesthesia, the patient was preoxygenated and thereafter induced with propofol $(150 \mathrm{mg})$ and ketamine $(30 \mathrm{mg})$ administered intravenously. Intubation was facilitated with rocuronium and anesthesia was maintained with sevoflurane and a total of $150 \mathrm{mcg}$ of fentanyl. No opioid narcotics or other analgesics were used intraoperatively. After 1.5 hours of an uneventful procedure, the patient was reversed, extubated, and transferred to the post-anesthesia care unit (PACU). Before emergence from anesthesia (secondary to the patient's refusal to place the block preoperatively), a peripheral nerve catheter (Pajunk E-Cath, Pajunk Medical Systems, Norcross, GA, US) was inserted perineurally at the right popliteal nerve using real-time ultrasound-guided visualization, followed by a bolus injection of 20 cc of $0.5 \%$ ropivacaine. An infusion of $0.2 \%$ ropivacaine was started after the initial bolus of local anesthetics. The infusion was delivered by an ambulatory infusion pump (Nimbus II PainPro, Infutronix Solutios LLC, Natick, MA, US) at $6 \mathrm{~mL} / \mathrm{hr}$. The patient required $15 \mathrm{mg}$ PO oxycodone in the PACU and was discharged the same day with minimal pain (2/10 numerical pain score). The follow-up call the next day revealed that the patient was satisfied with her pain management (pain 5/10 on the numeric scale) and was instructed to continue infusion until the pump reservoir became empty. No additional complaints about worsening neurological status were obtained the next day or on a subsequent follow-up visit six weeks after the procedure.

\section{Genotyping}

Because of the patient's history of erythromelalgia with an uncertain origin, we performed post hoc sequencing and genetic analysis of the total exome by next-generation sequencing. Blood deoxyribonucleic acid (DNA) was extracted, purified, and exome captured using the Agilent SureSelect Human All Exon V6 Kit (Agilent Technologies, CA, US). The whole exome sequencing strategy involved the creation of the 180-280 bp insert DNA library and sequencing on the HighSeq Illumina sequencer platform (Illumina, San Diego, CA, US) with an effective sequencing depth above 100×. The digital analysis pipeline of the raw data involved quality control and subsequent GATK-recommended strategy consisting of alignment sequence data (consisting of FASTQ files) with the reference genome (Hg19 and b37) using the GATK-Lite Variant Caller (Unified Genotyper from the GATK-like toolkit ver. 2.3;

www.broadinstitute.org/gatk/gatkdocs/org_broadinstitute_sting_gatk_walkers_genotyper_UnifiedGenotyper.ht and run on DNAnexus platform (platform.dnanexus.com/). This application calls SNPs and/or indels in the input sequence files, which were further annotated with wANNOVAR (wannovar.wglab.org). In addition, the presence of complex variant and copy number variants of the exome was further examined using Freebayes variant caller and CVNator on the same DNAnexus platform [3-6]. The presence of two alleles for this SNP in the investigated DNA sample was further verified employing the real-time TaqMan real-time polymerase chain reaction (PCR) method using the commercial kit (C_29261054_10) from ThermoFisher Scientific (Waltham, MA, US), according to the manufacturer's instructions.

The results of the analysis focused on the exonic loci for the sodium-channel genes, especially the SCN9A gene, which are listed in Table 1 . We identified 19 SNPs (nine nonsynonymous and 10 synonymous) in the loci containing voltage-gated sodium channel genes SCN1A - SCN11A, all present in the most current dbSNP database. We did not observe any of the previously reported mutations associated with erythromelalgia [79]. We noticed, however, that the patient was a homozygous carrier of missense and potentially damaging polymorphism rs6746030 in SCN9A, which was verified by the independent real-time TaqMan real-time PCR assay. All other SNPs in the investigated channels were either synonymous or were classified as benign. 


\section{Cureus}

\begin{tabular}{|c|c|c|c|c|c|c|c|}
\hline Chrom\# & Alt allele & Ref allele & Gene & SNP type & Position of codon and protein change & dbSNP & Carrier status \\
\hline 3 & $\mathrm{~T}$ & C & SCN10A & nonsynonymous & NM_001293307:exon26:c.A4843G:p.M1615V & rs6599241 & $1 / 1$ \\
\hline 3 & A & G & SCN10A & synonymous & NM_001293307:exon26:c.T4572C & rs6599242 & $1 / 1$ \\
\hline 3 & A & G & SCN11A & synonymous & NM_014139:exon12:c.T1638C & rs4073113 & $1 / 1$ \\
\hline 2 & $\mathrm{C}$ & $\mathrm{T}$ & SCN1A & nonsynonymous & NM_001165963:exon16:c.G3199A:p.A1067T & rs2298771 & $1 / 1$ \\
\hline 2 & A & G & SCN1A & synonymous & NM_001165963:exon13:c.T2292C & rs6432860 & $1 / 1$ \\
\hline 2 & $\mathrm{~T}$ & C & SCN1A & synonymous & NM_001165963:exon9:c.A1212G & rs7580482 & $1 / 1$ \\
\hline 19 & $\mathrm{~T}$ & $\mathrm{C}$ & SCN1B & nonsynonymous & NM_199037:exon3:c.T629C:p.L210P & rs557442440 & 0/1 \\
\hline 11 & G & A & SCN3B & synonymous & NM_018400:exon3:c.C438T & rs1275085 & $0 / 1$ \\
\hline 17 & $\mathrm{~T}$ & C & SCN4A & synonymous & NM_000334:exon24:c.A4869G & rs2070720 & $0 / 1$ \\
\hline 17 & $\mathrm{~T}$ & $\mathrm{C}$ & SCN4A & nonsynonymous & NM_000334:exon23:c.A4126G:p.N1376D & rs2058194 & $0 / 1$ \\
\hline 17 & $\mathrm{~T}$ & $\mathrm{C}$ & SCN4A & nonsynonymous & NM_000334:exon10:c.A1570G:p.S524G & rs6504191 & $1 / 1$ \\
\hline 3 & $\mathrm{~T}$ & $\mathrm{C}$ & SCN5A & synonymous & NM_000335:exon17:c.A3183G & rs7430407 & $1 / 1$ \\
\hline 3 & $\mathrm{~T}$ & $\mathrm{C}$ & SCN5A & nonsynonymous & NM_000335:exon12:c.A1673G:p.H558R & rs1805124 & $0 / 1$ \\
\hline 3 & $\mathrm{~T}$ & $\mathrm{C}$ & SCN5A & synonymous & NM_000335:exon2:c.A87G & rs6599230 & $0 / 1$ \\
\hline 2 & C & A & SCN7A & nonsynonymous & NM_002976:exon18:c.G2874T:p.M958| & rs6738031 & $0 / 1$ \\
\hline 2 & G & $\mathrm{T}$ & SCN7A & nonsynonymous & NM_002976:exon2:c.C122A:p.T41N & rs7565062 & $0 / 1$ \\
\hline 2 & A & G & SCN9A & nonsynonymous & NM_002977:exon19:c.T3448C:p.W1150R & rs6746030 & $1 / 1$ \\
\hline 2 & A & G & SCN9A & synonymous & NM_002977:exon10:c.T1119C & rs13414203 & $0 / 1$ \\
\hline 2 & $\mathrm{C}$ & T & SCN9A & synonymous & NM_002977:exon2:c.G174A & rs6432901 & 0/1 \\
\hline
\end{tabular}

TABLE 1: Common SNPs observed in the exonic region of SCN1A - SCN11A alpha-subunits of the voltage-gated sodium channel genes of the investigated patient by next-generation whole exome sequencing

SNP: single nucleotide polymorphism

\section{Discussion}

The two main forms of erythromelalgia are the primary and secondary types. Myeloproliferative diseases, together with thrombocythemia, are responsible for approximately $20 \%$ of cases of secondary erythromelalgia. The pathological signs in patients with secondary erythromelalgia include arteriolar intimal proliferation with thrombotic occlusions secondary to platelet aggregation [1-2]. Secondary erythromelalgia is most commonly associated with blood dyscrasias, connective tissue disorders, and drug reactions. Secondary erythromelalgia can result from a number of diseases such as myeloproliferative disorders (with a 3\%-65\% prevalence in patients with myeloproliferative disorders, especially polycythemia vera and essential thrombocytosis), hypercholesterolemia, autoimmune disorders, small fiber peripheral neuropathy, Fabry's disease, mercury poisoning, mushroom poisoning, sciatica, and some medications, including bromocriptine, verapamil, and ticlopidine [1-2].

Primary erythromelalgia (OMIM 133020), which is idiopathic or genetic, is a very rare autosomal dominant disorder. It represents channelopathy that is caused by rare mutations present in the SCN9A gene, which 
codes for the voltage-gated sodium channel NaV1.7 $\alpha$ subunit. These channels are preferentially expressed in the nociceptive dorsal root ganglion (DRG) [7-9]. Genomic studies in humans have provided compelling evidence emphasizing that high-impact rare mutations in hNaV1.7 with gain-of-function have marked effects on the channel function and clinical phenotype of familial primary erythromelalgia syndrome. The role of these mutations in pain pathways has been previously described [9]. The mutations associated with primary erythromelalgia are not always inherited as de novo mutations have been described.

Common missense SNPs in the exons of the SCN9A gene have more subtle and quantitative effects than the major mutations mentioned above but may also significantly influence an individual's pain perception. A common polymorphism (rs6746030) was previously found in the intracellular loop between domains II and III, with the minor allele occurring at a frequency of $\sim 10 \%$. The polymorphism results in a change of positively charged arginine (R, associated with minor A allele) to non-polar tryptophan (W, associated with the wild-type $\mathrm{G}$ allele) amino acid at residue 1150 in the reference human NaV1.7 sequence (Figure 1). The minor A allele was associated with increased pain perception when assessed in people with sciatica, amputees with phantom limb pain, and patients with post-discectomy lumbar root pain [10-17]. In addition, both clinical and experimental pain studies have shown that the level of pain felt was correlated with the genotype. That is, those patients with the least pain were wild-type GG genotype, intermediate pain in the GA genotype, and the most pain in the AA genotype, suggesting allelic dose dependency. To examine the functional effects of the rs6746030 SNPs, the two alleles that alter the coding sequence of NaV1.7, resulting in either 1150R (encoded by wild-type G allele) or 1150W (encoded by the minor A allele), were separately transfected into HEK-293 cells. The minor A allele was found to impair slow inactivation at voltages close to resting membrane potential, which is predicted to increase NaV1.7 activity [18]. In a different study, 1150W induced a depolarizing shift in the voltage dependence of activation compared with 1150R, inducing hyperexcitability in small DRG neurons, consistent with the clinical phenotype described in Reimann et al. [10-18]. These data suggest that SNPs in SCN9A resulting in NaV1.7 genetic variants may alter human pain threshold levels (i.e. produce quantitative changes in pain phenotype), and this variant may contribute to enhanced pain in common conditions such as back pain and osteoarthritis.

\section{a subunit}

I II<smiles>[AlH]=[V]</smiles>

III

IV

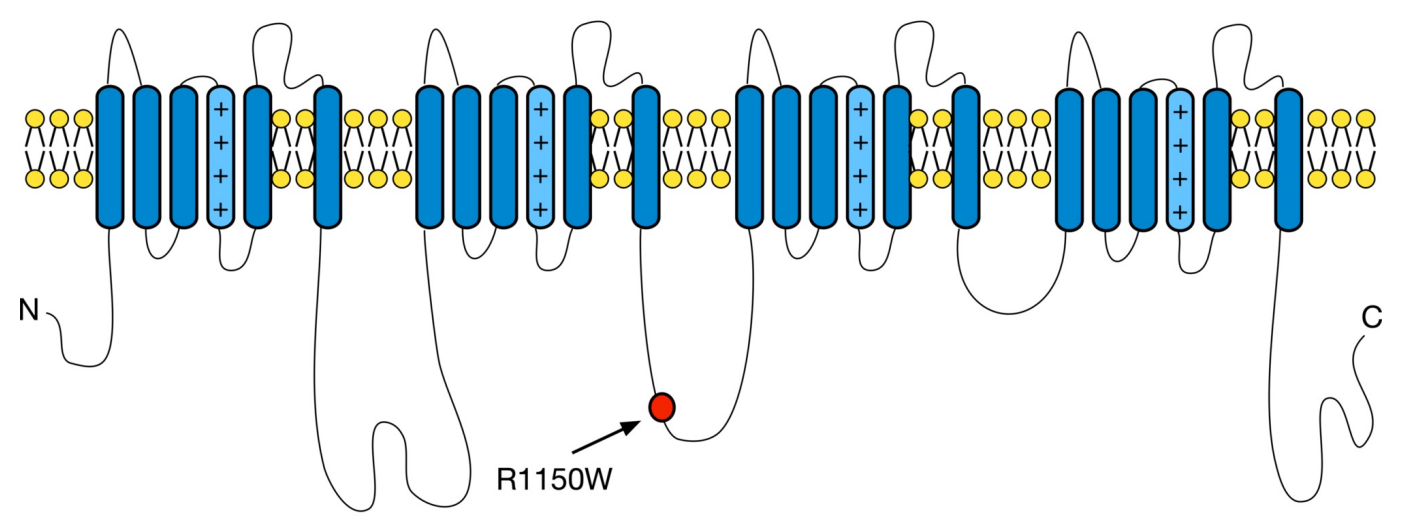

FIGURE 1: Nav1.7 alpha-subunit structure showing the location of amino acid affected by investigated common polymorphism rs 6746030 is marked with the arrow

Furthermore, Drenth et al. (2005) reported the same variant in the heterozygotic carrier and diagnosis of sporadic erythromelalgia. However, it was proposed that because the variant does not affect a highly conserved nucleotide sequence, it does not cause the phenotype but is rather an innocent sequence variation [8]. The authors suggested that this variant may cause a pain phenotype with low penetrance (in contrast to most familial types of primary erythromelalgia mutations, which exhibit nearly $100 \%$ penetrance), or that its phenotype expression may be modulated by disease-modifier gene(s). It should be noted that Estacion et al. (2009) showed electrophysiological evidence that in rats, small DRG neurons transiently transfected with $\mathrm{hNaV1.7}$ and that the polymorphism significantly increased the excitability of the neurons with the doubling of the firing frequency. In fact, the firing frequencies observed in DRG 
neurons transfected with the NaV1.7 1150W variant were similar to those seen in DRG neurons expressing the Q10R NaV1.7 mutant, which was found in a patient with primary erythromelalgia who had an onset of pain at 14 years, a greater age than typically observed in most patients with primary erythromelalgia [18]. It is unclear, in this respect, if the presence of two minor alleles of a frequent missense mutation in SCN9A rs6746030 - could also be associated with intractable chronic pain symptoms in sporadic (as in the reported case) erythromelalgia. It is not clear why the patient with the Q10R mutation did not experience pain earlier and why most humans carrying $1150 \mathrm{~W}$ NaV1.7 polymorphism do not develop chronic pain syndrome as primary erythromelalgia.

Because of the relative sparsity of homozygous rs6746030 carriers for the variant allele in the Caucasian population (3.29\% in Coriell database samples - as reported by Estacion et al. (2009) - or less than 1\% in our unpublished database), there are not many homozygous carriers of the variant allele to be investigated in cohort studies. Our observation suggests that the presence of two minor alleles in rs6746030 was related to intractable pain and erythromelalgia. In the majority of cases of secondary erythromelalgia reported so far, the chronic and tractable pain status is related to the degree of arteriolar inflammation, in which the platelet count is invariably significantly elevated. The patient in our report showed consistently normal levels of platelets. Similarly, this patient did not display signs of polycythemia vera and, in fact, the only one observed abnormality consisted of mild anemia and elevated red cells distribution width. It is also possible that our patient might represent the complex phenotype of mild erythromelalgia exacerbated by the presence of two variant alleles of the investigated polymorphism. Our observations in this study suggest the possibility that common missense polymorphism rs6746030 in NaV1.7 may not only contribute to the increased pain sensitivity or susceptibility to chronic pain but possibly, in at least some patients, contribute to symptoms of sporadic erythromelalgia.

The second interesting finding in this case report is the observed high efficiency of the peripheral continuous nerve block in managing the perioperative pain symptoms in the patient with erythromelalgia. Chronic pain symptoms in patients with erythromelalgia are very difficult to manage under conditions when a surgical procedure of the affected lower extremities is required. The evidence for or against performing peripheral nerve blocks in erythromelalgia is limited and not helpful because of concerns related to the exacerbation of underlying peripheral neuropathy [18]. Recently, Lorello et al. (2018) described the successful use of interscalene nerve block (single injection) as a complement to general anesthesia in a patient undergoing shoulder arthroplasty [19]. Thus, we reasoned that a similar approach of a continuous nerve block for the surgical procedure, along with a careful discussion of the benefits and risks with the patient, could be employed (for the first time reported in the literature for patients with erythromelalgia). Our results show that this approach led to the successful postoperative pain treatment with no neurological deficits. The six months follow-up (including neurological examination) did not find new neurological deficits or a worsening of erythromelalgia-associated pain status.

\section{Conclusions}

Chronic pain associated with erythromelalgia may be associated not only with the severe myelodysplasia and carrier status for rare variants but also with the homozygous carrier status for common missense polymorphism rs6746030 R1150W. Continuous nerve block in the perioperative period represents a safe and efficient modality for postoperative pain management in erythromelalgia patients.

\section{Additional Information \\ Disclosures}

Human subjects: Consent was obtained by all participants in this study. Conflicts of interest: In compliance with the ICMJE uniform disclosure form, all authors declare the following: Payment/services info: All authors have declared that no financial support was received from any organization for the submitted work. Financial relationships: All authors have declared that they have no financial relationships at present or within the previous three years with any organizations that might have an interest in the submitted work. Other relationships: All authors have declared that there are no other relationships or activities that could appear to have influenced the submitted work.

\section{References}

1. van Genderen PJ, Michiels JJ: Erythromelalgia: a pathognomonic microvascular thrombotic complication in essential thrombocythemia and polycythemia vera. Semin Thromb Hemost. 1997, 23:357-363. 10.1055/s2007-996109 
2. Kang BC, Nam DJ, Ahn EK, Yoon DM, Cho JG: Secondary erythromelalgia - a case report - . Korean J Pain. 2013, 26:299-302. 10.3344/kjp.2013.26.3.299

3. Yang H, Wang K: Genomic variant annotation and prioritization with ANNOVAR and wANNOVAR . Nat Protoc. 2015, 10:1556-1566. 10.1038/nprot.2015.105

4. Chang X, Wang K: wANNOVAR: annotating genetic variants for personal genomes via the web . J Med Genet. 2012, 49:433-436. 10.1136/jmedgenet-2012-100918

5. Wang K, Li M, Hakonarson H: ANNOVAR: functional annotation of genetic variants from high-throughput sequencing data. Nucleic Acids Res. 2010, 38:164. 10.1093/nar/gkq603

6. Yang H, Robinson PN, Wang K: Phenolyzer: phenotype-based prioritization of candidate genes for human diseases. Nature Methods. 2015, 12:841-843. 10.1038/nmeth.3484

7. Bennett DL, Clark AJ, Huang J, Waxman SG, Dib-Hajj SD: The role of voltage-gated sodium channels in pain signaling. Physiol Rev. 2019, 1:1079-1151. 10.1152/physrev.00052.2017

8. Drenth JP, te Morsche RH, Guillet G, Taieb A, Kirby RL, Jansen JB: SCN9A mutations define primary erythermalgia as a neuropathic disorder of voltage gated sodium channels. J Invest Dermatol. 2005, 124:1333-1338. 10.1111/j.0022-202X.2005.23737.x

9. Drenth JP, Waxman SG: Mutations in sodium channel gene SCN9A cause a spectrum of human genetic pain disorders. J Clin Invest. 2007, 11:3603-3609. 10.1172/JCI33297

10. Reimann F, Cox JJ, Belfer I, et al.: Pain perception is altered by a nucleotide polymorphism in SCN9A . Natl Acad Sci USA. 2010, 107:5148-5153. 10.1073/pnas.0913181107

11. Kurzawski M, Rut M, Dziedziejko V, Safranow K, Machoy-Mokrzynska A, Drozdzik M, Bialecka M: Common missense variant of SCN9A gene is associated with pain intensity in patients with chronic pain from disc herniation. Pain Med. 2018, 19:1010-1014. 10.1093/pm/pnx261

12. Duan G, Xiang G, Guo S, et al.: Genotypic analysis of SCN9A for prediction of postoperative pain in female patients undergoing gynecological laparoscopic surgery. Pain Physician. 2016, 19:151-162.

13. Duan G, Guo S, Zhang Y, et al.: The effect of SCN9A variation on basal pain sensitivity in the general population: an experimental study in young women. J Pain. 2015, 16:971-980. 10.1016/j.jpain.2015.06.011

14. Reeder JE, Byler TK, Foster DC, et al.: Polymorphism in the SCN9A voltage-gated sodium channel gene associated with interstitial cystitis/ bladder pain syndrome. Urology. 2013, 81:210.

10.1016/j.urology.2012.07.072

15. Holliday KL, Thomson W, Neogi T, et al.: The nonsynonymous SNP, R1150W, in SCN9A is not associated with chronic widespread pain susceptibility. Mol Pain. 2012, 8: 10.1186/1744-8069-8-72

16. Valdes AM, Arden NK, Vaughn FL, et al.: Role of the Nav1.7 R1150W amino acid change in susceptibility to symptomatic knee osteoarthritis and multiple regional pain. Arthritis Care Res (Hoboken). 2011, 63:440444. 10.1002/acr.20375

17. Estacion M, Harty TP, Choi JS, Tyrrell L, Dib-Hajj SD, Waxman SG: A sodium channel gene SCN9A polymorphism. Ann Neurol. 2009, 66:862-866. 10.1002/ana.21895

18. Neal JM, Bernards CM, Hadzic A, et al.: ASRA practice advisory on neurologic complications in regional anesthesia and pain medicine. Reg Anesth Pain Med. 2008, 33:404-415.

19. Lorello GR, Perlas A: Erythromelalgia and peripheral nerve block: a case report . A A Pract. 2019, 12:264266. 